\title{
Sum-Throughput Maximization in Backscatter Communication-Based Cognitive Networks
}

\author{
Qian Li iD \\ School of Computer Science and Technology, Zhoukou Normal University, Zhoukou, China \\ Correspondence should be addressed to Qian Li; liqian@zknu.edu.cn
}

Received 8 December 2021; Revised 2 January 2022; Accepted 11 January 2022; Published 4 February 2022

Academic Editor: Yinghui Ye

Copyright (C) 2022 Qian Li. This is an open access article distributed under the Creative Commons Attribution License, which permits unrestricted use, distribution, and reproduction in any medium, provided the original work is properly cited.

\begin{abstract}
In this paper, we consider a backscatter communication (BackCom) based cognitive network that consists of one primary transmitter, one primary receiver, multiple secondary transmitters (STs), and one secondary receiver (SR). Each ST operates in the BackCom or energy harvesting model. Our goal is to jointly optimize the energy harvesting and backscatter time, the transmit power of the primary transmitter, and the power reflection coefficient of each ST to maximize the sum throughput of all the STs under a nonlinear energy harvesting model while satisfying multiple constraints, i.e., the energy causality of each ST, the Quality of Service of the primary transmitter, etc. The formulated problem is nonconvex due to the coupled variables and hard to solve. In order to address this problem, we decouple partial coupled variables by using the properties of the objective function and constructing auxiliary variables, and the remaining coupled variables are decoupled via successive convex approximation (SCA). On this basis, a SCA based iterative algorithm is developed to solve the formulated problem. Simulation results are provided to support our work.
\end{abstract}

\section{Introduction}

Internet of Things (IoT) is expected to deploy massive smart sensor nodes in the future communications to seamlessly connect the physical environment and the cyberspace for providing intelligent services [1]. However, such an approach requires huge spectrum resources, and this motivates us to consider high spectrum-efficient communication paradigms for IoT. In this context, cognitive radio has been proposed, whose key idea is to allow sensor nodes sharing spectrum with primary uses without causing any harmful factors to the primary transmission [2]. In cognitive radio, the smart sensor node (also called secondary user) transmits its own information by active radios that need the powerconsuming components and consume a lot of energy, greatly shortening their lifespan and leading to an energy-constrained problem for smart sensor nodes.

In addition to the cognitive radio that improves the spectrum efficiency, backscatter communication (BackCom) is another key technology, and its main purpose is to overcome the energy-constrained problem [3-5]. BackCom allows a smart sensor node modulating its information on the incident signals and backscattering the modulated signals to its associated receiver by changing the power reflection coefficient so that the power-consuming components can be avoided, while harvesting energy from the incident signal for realizing energy self-sustainability [3-5]. Despite these superiorities, the communication performance of BackCom is limited as it uses the ambient signals as the incident signals, and the ambient signals introduce serious cochannel interference to the BackCom receiver [4]. Accordingly, researchers proposed to use the controllable signals as the incident signals, but such an approach requires an extra cost to deploy RF sources. Recall that cognitive radio is able to provide controllable signals for the BackCom transmitter (also referred to as the secondary transmitter (ST) in this paper). Recent works have integrated BackCom into cognitive radio, yielding a spectrum- and energy-efficiency paradigm, called BackCom based cognitive networks.

In [6], the authors formulated a problem to maximize the throughput of a BackCom link in a BackCom based cognitive network with a single ST by jointly optimizing the transmit 
power of the primary user and the power reflection coefficient of the ST. In [7], the authors proposed to maximize the energy efficiency of the secondary link by jointly optimizing the transmit power of the primary transmitter (PT), the power reflection coefficient of the ST, and the time for energy harvesting and BackCom. Extending the single ST scenario $[6,7]$ into multiple STs [8], the authors maximized the sum rate of STs by jointly optimizing the PT's transmit power and the power reflection coefficient of each ST. The authors of [9] considered a full-duplex-enabled BackCom based cognitive network and proposed a joint time, transmit power, and power reflection coefficient scheduling to maximize the throughput of the BackCom system. In addition to the above works, the harvest-then-transmit (HTT) protocol has also been integrated into BackCom based cognitive networks, and various resource allocation schemes have been studied [10-12], where the main focus is to balance the time for energy harvesting, HTT, and backscattering.

After carefully examining the existing resource allocation schemes, we note that all of them were based on a linear energy harvesting model. As pointed out by existing works, the linear energy harvesting model does not match the behavior of a practical energy harvester. More specifically, the harvest power is a nonlinear function with respect to the input power. The previous works [13-15] (in which BackCom based cognitive network has not been considered) have proved that the mismatch of energy harvesting models will lead to performance degradation. Accordingly, it is necessary to design resource allocation for BackCom based cognitive networks with nonlinear energy harvesting model. Motivated by this, in this paper, we consider a BackCom based cognitive network with multiple STs and aim to maximize the total throughput of STs by jointly optimizing the energy harvesting time, BackCom time, power reflection coefficient, and PT's transmit power under a nonlinear energy harvesting model. Meanwhile, the energy-causality constraint of each ST and the Quality of Service (QoS) of both the primary link and the secondary links are considered. The main contributions of this paper are summarized as below.

We formulate an optimization problem to maximize the sum throughput of STs, and propose an efficient iterative algorithm to solve the problem. Since the formulated problem is nonconvex, the main challenge is how to transform the original problem into a convex one. Towards this end, we firstly determine the optimal transmit power of the PT by using the properties of the objective function, then introduce some auxiliary variables to decouple coupled variables, and lastly employ the successive convex approximation (SCA) to transform a nonconvex constraint into a linear one. We also provide computer simulation results to verify the proposed iterative algorithm and show the advantages of the proposed scheme over the baseline schemes.

\section{System Model and Working Flow}

As shown in Figure 1, we consider a BackCom based cognitive network, which consists of one PT, one PR, $K$ energy-constrained STs, and one SR. In this network, the PT

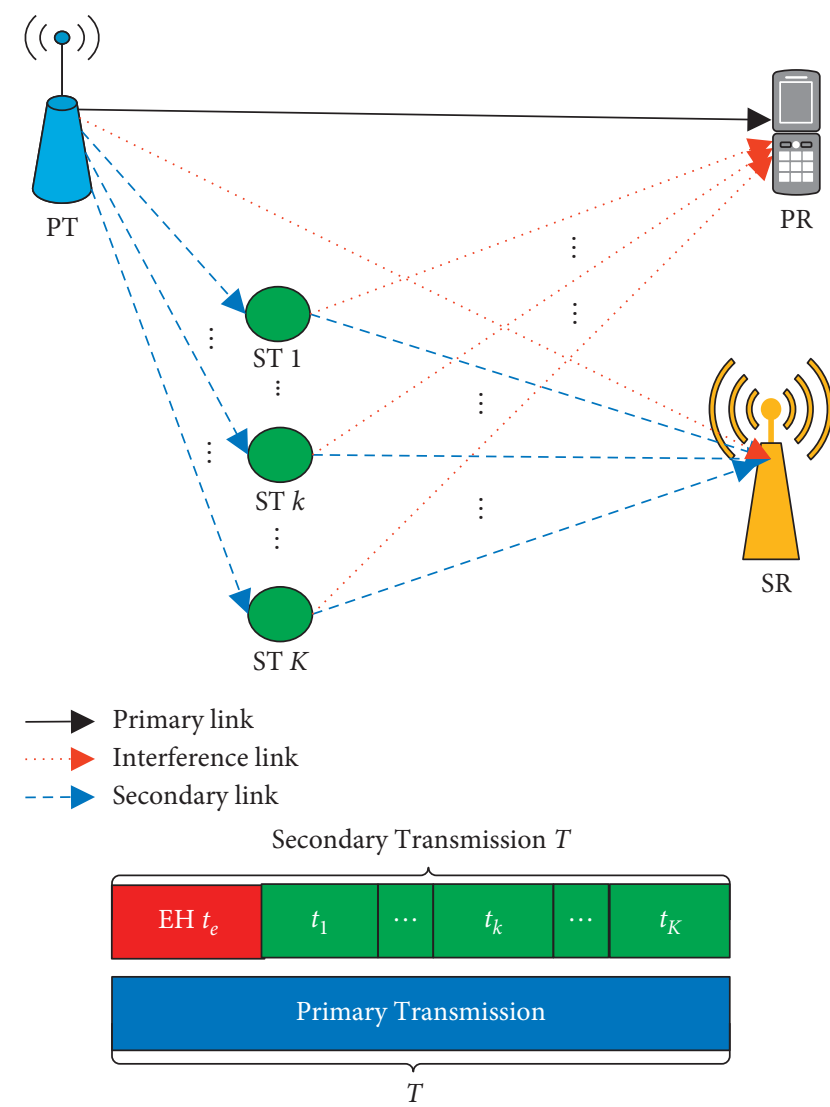

Figure 1: BackCom based cognitive network and its frame structure.

transmits its own information to the PR, while the RF signals transmitted by the PT can also be exploited by the $K$ STs for energy harvesting (EH) and information transmission. Assume that all the devices are equipped with a single antenna and work in the half-duplex mode. Suppose that each ST is equipped with both the EH circuit and the BackCom circuit so that it can harvest energy from the received RF signals and backscatter the received signals for information transmission. In order to prolong the operation time of each energy-constrained ST, we assume that each ST only uses its harvested energy instead of the energy early stored in its battery to cover the energy consumption during information backscattering. All channels including the PT-PR link and the ST-SR links are assumed to follow quasi-static fading. In the beginning of each transmission block, the channel state information (CSI) of all links can be perfectly achieved by the PT via the existing advanced channel estimation methods, so that the PT can determine the optimal resource allocation scheme based on the obtained CSI and feed the optimal scheme back to all the STs.

Let $T$ denote the duration of the whole transmission block. For the PT-PR link, the PT may transmit its signals in the whole transmission block. For the ST-SR links, the whole transmission can be divided into two phases, which are the $\mathrm{EH}$ phase and the BackCom phase, respectively. In the $\mathrm{EH}$ phase, the PT broadcasts its signals to the PR while all the STs will perform $\mathrm{EH}$. In the BackCom phase, the PT keeps 
broadcasting while all the STs take turns to perform BackCom in order to avoid the cochannel interference among STs.

2.1. EH Phase. Let $t_{\mathrm{e}}$ denote the duration of the $\mathrm{EH}$ phase. Denote $P_{\mathrm{t}}$ as the transmit power of the PT. Then, the received signal at the $k$-th $(k \in \mathscr{K}=\{1,2, \ldots, K\}) \mathrm{ST}$ is given by

$$
y_{\mathrm{ST}}^{k}=\sqrt{P_{\mathrm{t}} h_{k}} x_{p}+N_{\mathrm{ST}},
$$

where $h_{k}$ denotes the channel gain of the PT- $k$-th ST link, $x_{p}$ with $\mathbb{E}\left[\left|x_{p}\right|^{2}\right]=1$ is the information transmitted by the PT to the PR, and $N_{\mathrm{ST}}$ is the thermal noise at the $k$-th ST. Since the backscatter communication circuit consists only of passive components and takes few signal processing operations, the thermal noise is usually very small and can be ignored, i.e., $N_{\mathrm{ST} \approx 0}$ [7].

For $\mathrm{EH}$, we consider a more practical nonlinear $\mathrm{EH}$ model since the linear $\mathrm{EH}$ model cannot characterize the nonlinearity of the practical EH circuit [16]. The reasons of considering the above nonlinear $\mathrm{EH}$ model instead of the one considered in $[17,18]$ are as follows. Firstly, the nonlinear EH model proposed in [16] is accurate enough for characterizing the nonlinearity of practical $\mathrm{EH}$ circuits. Secondly, the use of the nonlinear EH model proposed in [16] can simplify the difficulty and reduce the complexity of solving the formulated optimization problem. Accordingly, we compute the harvested energy of the $k$-th as

$$
E_{\mathrm{e}}^{k}=t_{\mathrm{e}}\left(\frac{a_{k} P_{\mathrm{t}} h_{k}+d_{k}}{P_{\mathrm{t}} h_{k}+v_{k}}-\frac{d_{k}}{v_{k}}\right)
$$

where $a_{k}, d_{k}$, and $v_{k}$ are the parameters of the nonlinear EH model.

For the PT-PR link, the received signal at the PR is expressed as

$$
y_{\mathrm{PR}}^{e}=\sqrt{P_{\mathrm{t}} f_{p}} x_{p}+N_{\mathrm{PR}}
$$

where $f_{p}$ denotes the channel gain of the PT-PR link and $N_{\text {PR }}$ is the additive white Gaussian noise (AWGN) at the PR with mean zero and variance $\sigma^{2}$. Accordingly, the achievable throughput at the PR in this phase can be computed as

$$
R_{\mathrm{e}}^{p}=t_{\mathrm{e}} B \log _{2}\left(1+\frac{P_{\mathrm{t}} f_{p}}{\sigma^{2}}\right),
$$

where $B$ is the system bandwidth.

2.2. BackCom Phase. The whole BackCom phase can be divided into $K$ subphases. In each subphase, each ST performs BackCom to transmitted information. Let $t_{k}$ denote the duration of the $k$-th subphase. Denote $\alpha_{k}$ with $0 \leq \alpha_{k} \leq 1$ as the power reflection coefficient of the $k$-th ST based on which the received RF signal at the $k$-th ST can be split into two parts: one part is used for BackCom and the other part is flowed into the EH circuit. Then, in the subphase $t_{k}$, the received signal at the $\mathrm{SR}$ is given by

$$
y_{\mathrm{SR}}^{k}=\sqrt{\alpha_{k} P_{\mathrm{t}} h_{k} g_{k}} x_{p} x_{s, k}+\sqrt{P_{\mathrm{t}} f_{s}} x_{p}+N_{\mathrm{SR}}
$$

where $g_{k}$ denotes the channel gain between the $k$-th ST and the SR, $f_{s}$ is the channel gain of the PT-SR link, $x_{s, k}$ with $\mathbb{E}\left[\left|x_{s, k}\right|^{2}\right]=1$ is the transmitted information of the $k$-th ST, and $N_{\mathrm{SR}}$ is the $\mathrm{AWGN}$ at the $\mathrm{SR}$ with mean zero and variance $\sigma^{2}$.

From (5), it can be observed that the cochannel interference from the PT-SR link always exists, which degrades the throughput achieved by the $k$-th ST via BackCom. In order to decode the transmitted information of the $k$-th ST correctly, the SR performs the successive interference cancellation (SIC) technology. Specifically, the SR first decodes the PT's transmitted information $x_{p}$ by treating $\sqrt{\alpha_{k} P_{\mathrm{t}} h_{k} g_{k}} x_{p} x_{s, k}$ as the cochannel interference and then uses the SIC technology to cancel the interference from the PT as well as decoding the transmitted information of the $k$-th ST $x_{s, k}$. Therefore, the signal to interference plus noise ratio (SINR) at the SR for decoding $x_{p}$ is given by

$$
\gamma_{s, k}^{p}=\frac{P_{\mathrm{t}} f_{s}}{\alpha_{k} P_{\mathrm{t}} h_{k} g_{k}+\sigma^{2}} .
$$

After using SIC technology, the signal to noise ratio (SNR) at the SR for decoding $x_{s, k}$ is expressed as

$$
\gamma_{s, k}=\frac{\alpha_{k} P_{\mathrm{t}} h_{k} g_{k}}{\eta P_{\mathrm{t}} f_{s}+\sigma^{2}}
$$

where $\eta$ with $0 \leq \eta \leq 1$ is the interference cancellation factor.

Accordingly, the achievable throughput of the $k$-th STSR link can be computed as

$$
R_{s, k}=t_{k} B \log _{2}\left(1+\xi \gamma_{s, k}\right)
$$

where $\xi$ expresses the performance gap reflecting the real modulation [19-21]. In this subphase, the harvested energy of the $k$-th ST is determined by

$$
E_{k}^{\mathrm{b}}=t_{k}\left(\frac{a_{k}\left(1-\alpha_{k}\right) P_{\mathrm{t}} h_{k}+d_{k}}{\left(1-\alpha_{k}\right) P_{\mathrm{t}} h_{k}+v_{k}}-\frac{d_{k}}{v_{k}}\right) \text {. }
$$

At the end of the BackCom phase, the total harvested energy of the $k$-th ST is given by

$$
E_{\mathrm{tot}}^{k}=E_{k}^{\mathrm{b}}+\left(t_{\mathrm{e}}+\sum_{i=1}^{K} t_{i}-t_{k}\right)\left(\frac{a_{k} P_{\mathrm{t}} h_{k}+d_{k}}{P_{\mathrm{t}} h_{k}+v_{k}}-\frac{d_{k}}{v_{k}}\right) .
$$

For the PT-PR link, it also suffers from the cochannel interference from the $k$-th ST, and the received signal at the $\mathrm{PR}$ is given by

$$
y_{\mathrm{PR}}^{k}=\sqrt{P_{\mathrm{t}} f_{p}} x_{p}+\sqrt{\alpha_{k} P_{\mathrm{t}} h_{k} f_{k}} x_{p} x_{s, k}+N_{\mathrm{PR}},
$$

where $f_{k}$ denotes the channel gain from the $k$-th ST to the $\mathrm{PR}$. Then, the SINR at the PR for decoding $x_{p}$ is computed as

$$
\gamma_{k}^{p}=\frac{P_{\mathrm{t}} f_{p}}{\alpha_{k} P_{\mathrm{t}} h_{k} f_{k}+\sigma^{2}} .
$$


Correspondingly, the achievable throughput of the PT$\mathrm{PR}$ link in this subphase is given by $R_{k}^{p}=t_{k} B \log _{2}\left(1+\gamma_{k}^{p}\right)$.

\section{Throughput Maximization for BackCom- Based Cognitive Networks}

In this section, we design an optimal resource allocation scheme to maximize the total throughput of all the STs for the BackCom based cognitive network. In particular, we formulate a throughput maximization problem by jointly optimizing the transmit power of the PT, BackCom time, and power reflection coefficients of STs, as well as the EH time, subject to QoS, energy causality, latency, transmit power, and power reflection coefficient constraints, and then use the existing convex tools to solve it.

3.1. Problem Formulation. Before formulating the throughput maximization problem, we should determine the optimization objective and constraints.

3.1.1. Optimization Objective. The optimization objective is to maximize the total throughput of the STs which can be computed as

$$
R_{\text {tot }}^{s}=\sum_{k=1}^{K} R_{s, k}=\sum_{k=1}^{K} t_{k} B \log _{2}\left(1+\frac{\xi \alpha_{k} P_{\mathrm{t}} h_{k} g_{k}}{\eta P_{\mathrm{t}} f_{s}+\sigma^{2}}\right) .
$$

3.1.2. QoS Constraints. There are two QoS constraints to constrain the throughput of the PT and each ST, respectively. For the QoS constraint of each ST, we should guarantee that the achievable throughput of each ST is not less than its minimum required throughput. Let $C_{\min , k}$ denote the minimum required throughput of the $k$-th ST. Then, the QoS constraint of the $k$-th ST can be expressed as

$$
\begin{aligned}
R_{s, k} & \geq C_{\min , k}, \quad \forall k, \\
\Longleftrightarrow t_{k} B \log _{2}\left(1+\frac{\xi \alpha_{k} P_{\mathrm{t}} h_{k} g_{k}}{\eta P_{\mathrm{t}} f_{s}+\sigma^{2}}\right) & \geq C_{\min , k}, \quad \forall k .
\end{aligned}
$$

For the QoS constraint of the PT, the total achievable throughput of the PT should not be less than the PT's minimum required throughput, denoted by $C_{\min }$. Therefore, the QoS constraint of the PT can be expressed as

$$
\begin{aligned}
R_{e}^{p}+\sum_{k=1}^{K} R_{k}^{p}= & t_{e} B \log _{2}\left(1+\frac{P_{t} f_{p}}{\sigma^{2}}\right) \\
& +\sum_{k=1}^{K} t_{k} B \log _{2}\left(1+\frac{P_{t} f_{p}}{\alpha_{k} P_{t} h_{k} f_{k}+\sigma^{2}}\right) \geq C_{\min } .
\end{aligned}
$$

3.1.3. Energy-Causality Constraint. The energy-causality constraint states that the energy consumption of each ST should not be larger than its harvested energy during the whole transmission block. Note that a rechargeable battery is equipped in each ST and that each ST may first use the energy stored in its battery to support BackCom and then use the harvested energy to power the battery. The energy-causality constraint ensures that the energy early stored in the battery is not reduced. Here, we consider fixed power consumption for BackCom by following [19-21]. Let $P_{\mathrm{b}, k}$ denote the power consumption for the $k$-th ST when performing BackCom. Therefore, the energy-causality constraint for the $k$-th ST is given by

$$
\begin{aligned}
P_{b, k} t_{k} \leq & E_{\text {tot }}^{k}=t_{k} f_{k}\left(\left(1-\alpha_{k}\right) P_{t} h_{k}\right) \\
& +\left(t_{e}+\sum_{i=1}^{K} t_{i}-t_{k}\right) f_{k}\left(P_{t} h_{k}\right), \quad \forall k,
\end{aligned}
$$

where $f_{k}(x)=\left(a_{k} x+d_{k} / x+v_{k}\right)-\left(d_{k} / v_{k}\right)$. Please note that the power consumption for the $\mathrm{EH}$ circuit has been included in the EH model and thus has not been considered in (16).

3.1.4. Transmit Power Constraint. Let $P_{\max }$ denote the maximum allowed transmit power of the PT. Then, the PT's transmit power constraint can be expressed as

$$
0 \leq P_{\mathrm{t}} \leq P_{\max } .
$$

Based on (13), (14), (15), (16), and (17), the throughput maximization problem can be formulated as

$$
\begin{aligned}
& \mathrm{P}_{1}: \underset{P_{t}, t_{e},\left\{t_{k}\right\}_{k=1}^{K},\left\{\alpha_{k}\right\}_{k=1}^{K}}{s} R_{t o t}^{s}, \\
& \text { s.t. C1: }(14),(15), \\
& \text { C2: }(16), \\
& \text { C3: }(17), \\
& \text { C4: }\left(\gamma_{s, k}^{p} \geq \gamma_{t h}, \forall k\right), \\
& \text { C5: } t_{e}+\sum_{k=1}^{K} t_{k} \leq T, t_{e}, t_{k} \geq 0, \forall k, \\
& \text { C6: } 0 \leq \alpha_{k} \leq, \forall k,
\end{aligned}
$$

where $\mathrm{C} 1$ denotes the QoS constraints for each ST and the PT, C2 is the energy-causality constraint for each ST, C3 constrains the maximum transmit power of the PT, C4 ensures that each ST can decode $x_{p}$ successfully and $\gamma_{\text {th }}$ is the threshold required for decoding $x_{p}$, C5 is the latency constraint, and C6 is the constraint for the power reflection coefficient of each ST.

It can be observed that $\mathbf{P}_{1}$ is a highly nonconvex optimization problem and is difficult to solve due to the following reasons. Firstly, there exist several coupled relationships between multiple optimization variables, i.e., $P_{\mathrm{t}}, t_{k}, \alpha_{k}$, etc., leading to the nonconvex objective function and constraints, i.e., C1, C2, etc. Secondly, the cochannel interference causes the difference of convex (DC) structures in the objective function and $\mathrm{C} 1$, bringing new challenges to solving $\mathbf{P}_{1}$. Thirdly, the consideration of the nonlinear $\mathrm{EH}$ model is another difficulty for solving $\mathbf{P}_{1}$ since the nonlinear $\mathrm{EH}$ model makes C1 more complex. Therefore, it is hard to solve $\mathbf{P}_{1}$. 
3.2. Solution. In order to remove DC structures existing in the objective function and $\mathrm{C} 1$ and simplify $\mathbf{P}_{1}$, the following lemma is introduced to determine the optimal transmit power of the PT.
Lemma 1. The maximum throughput of all the STs for the considered network is achieved when the PT transmits its signals with its maximum transmit power, i.e., $P_{t}^{*}=P_{\max }$.

Proof. Please see Appendix A.

By substituting $P_{\mathrm{t}}=P_{\max }$ into $\mathbf{P}_{1}, \mathbf{P}_{1}$ can be reformulated as

$$
\begin{aligned}
& \mathrm{P}_{2}: \max _{t_{e},\left\{t_{k}\right\}_{k=1}^{K},\left\{\alpha_{k}\right\}_{k=1}^{K}} \sum_{k=1}^{K} t_{k} B \log _{2}\left(1+\frac{\zeta \alpha k P_{\max } h_{k} g_{k}}{\eta P_{\max } f_{s}+\sigma^{2}}\right), \\
& \text { s.t. } C 1^{\prime}: t_{k} B \log _{2}\left(1+\frac{\zeta \alpha k P_{\max } h_{k} g_{k}}{\eta P_{\max } f_{s}+\sigma^{2}}\right) \geq C_{\min , k}, \forall k, \\
& \sum_{k=1}^{K} t_{k} B \log _{2}\left(1+\frac{P_{\max } f_{p}}{\alpha_{k} P_{\max } h_{k} f_{k}+\sigma^{2}}\right)+t_{e} B \log _{2}\left(1+\frac{P_{\max } f_{p}}{\sigma^{2}}\right) \geq C_{\min }, \\
& C 2^{\prime}: P_{b, k} t_{k} \leq\left(t_{e}+\sum_{i=1}^{K} t_{i}-t_{k}\right) f_{k}\left(P_{\max } h_{k}\right)+t_{k} f_{k}\left(\left(1-\alpha_{k}\right) P_{\max } h_{k}\right), \forall k, \\
& C 5, C 7: 0 \leq \alpha_{k} \leq \min \left(\frac{P_{\max } f_{s}-\gamma_{t h} \sigma^{2}}{P_{\max } h_{k} g_{k} \gamma_{t h}}, 1\right), \forall k,
\end{aligned}
$$

where $\mathrm{C} 7$ is the combination of $\mathrm{C} 4$ and C6.

$\mathbf{P}_{2}$ is still nonconvex since the coupled relationships between different variables, e.g., $\alpha_{k}$ and $t_{k}$, still exist in the objective function and several constraints. To address this issue, we introduce the following auxiliary variables: $z_{k}=\alpha_{k} t_{k}, \forall k$, to replace the variables $\alpha_{k}, \forall k$, and rewrite $\mathbf{P}_{2}$ as

$$
\begin{aligned}
& \mathrm{P}_{2}: \max _{t_{e},\left\{t_{k}\right\}_{k=1}^{K},\left\{z_{k}\right\}_{k=1}^{K}} \sum_{k=1}^{K} t_{k} B \log _{2}\left(1+\frac{\zeta z_{k} P_{\max } h_{k} g_{k}}{t_{k}\left(\eta P_{\max } f_{s}+\sigma^{2}\right)}\right), \\
& \text { s.t. } C 1^{\prime \prime}: t_{k} B \log _{2}\left(1+\frac{\zeta z_{k} P_{\max } h_{k} g_{k}}{t_{k}\left(\eta P_{\max } f_{s}+\sigma^{2}\right)}\right) \geq C_{\min , k}, \forall k, \\
& \sum_{k=1}^{K} t_{k} B \log _{2}\left(1+\frac{P_{\max } f_{p}}{z_{k} P_{\max } h_{k} f_{k}+\sigma^{2}}\right)+t_{e} B \log _{2}\left(1+\frac{P_{\max } f_{p}}{\sigma^{2}}\right) \geq C_{\min }, \\
& C 2^{\prime \prime}: P_{b, k} t_{k} \leq t_{k} f_{k}\left(\frac{\left(t_{k}-z_{k}\right) P_{\max } h_{k}}{t_{k}}\right)\left(t_{e}+\sum_{i=1}^{K} t_{i}-t_{k}\right) f_{k}\left(P_{\max } h_{k}\right), \forall k, \\
& C 5, C 7^{\prime}: 0 \leq z_{k} \leq t_{k} \times \min \left(\frac{P_{\max } f_{s}-\gamma_{t h} \sigma^{2}}{P_{\max } h_{k} g_{k} \gamma_{t h}}, 1\right)
\end{aligned}
$$

where $\alpha_{k}=\left(z_{k} / t_{k}\right), \forall k$.

Proposition 1. In $\mathbf{P}_{3}$, the objective function and all the constraints except $C 1^{\prime \prime}$ are convex.

Proof. Please see Appendix B. 
In order to handle the nonconvex constraint $\mathrm{C}^{\prime \prime}$ and solve $\mathbf{P}_{3}$, the SCA method is used, where the first-order Taylor expression is used to approximate the function $t_{k} B \log _{2}\left(1+\left(t_{k} P_{\max } f_{p} / z_{k} P_{\max } h_{k} f_{k}+\sigma^{2} t_{k}\right)\right)$ in $\mathrm{C} 1^{\prime \prime}$ and turn this nonconvex function into a linear function. Specifically, let $F_{k}\left(\alpha_{k}\right)=t_{k} B \log _{2}\left(1+\left(P_{\max } f_{p} / \alpha_{k} P_{\max }\right.\right.$ $\left.\left.h_{k} f_{k}+\sigma^{2}\right)\right)$. By taking the first-order derivative of $F_{k}\left(\alpha_{k}\right)$ with respect to $\alpha_{k}$, we have

$$
\frac{\partial F_{k}\left(\alpha_{k}\right)}{\partial \alpha_{k}}=\frac{-P_{\max ^{2}} f_{p} h_{k} f_{k} B t_{k}}{\left(\alpha_{k} P_{\max } h_{k} f_{k}+\sigma^{2}+P_{\max } f_{p}\right)\left(\alpha_{k} P_{\max } h_{k} f_{k}+\sigma^{2}\right) \operatorname{In} 2} .
$$

Using the first-order Taylor expression, $F_{k}\left(\alpha_{k}\right)$ can be approximated as

$$
\begin{aligned}
F_{k}\left(\alpha_{k}\right) \approx \frac{\partial F_{k}\left(\alpha_{k}^{0}\right)}{\partial \alpha_{k}^{0}}\left(\alpha_{k}-\alpha_{k}^{0}\right)+F_{k}\left(\alpha_{k}^{0}\right) & =\frac{-P_{\max ^{2}} f_{p} h_{k} f_{k} B t_{k}\left(\alpha_{k}-\alpha_{k}^{0}\right)}{\left(\alpha_{k}^{0} P_{\max } h_{k} f_{k}+\sigma^{2}+P_{\max } f_{p}\right)\left(\alpha_{k}^{0} P_{\max } h_{k} f_{k}+\sigma^{2}\right) \operatorname{In} 2}+F_{k}\left(\alpha_{k}^{0}\right), \\
& =\frac{-P_{\max ^{2}} f_{p} h_{k} f_{k} B\left(z_{k}-\alpha_{k}^{0} t_{k}\right)}{\left(\alpha_{k}^{0} P_{\max } h_{k} f_{k}+\sigma^{2}+P_{\max } f_{p}\right)\left(\alpha_{k}^{0} P_{\max } h_{k} f_{k}+\sigma^{2}\right) \operatorname{In} 2}+F_{k}\left(\alpha_{k}^{0}\right),
\end{aligned}
$$

where $\alpha_{k}^{0}$ is the given value for $\alpha_{k}$ and can be updated iteration by iteration.
By substituting (22) into C1", the QoS constraint for the PT's transmission can be rewritten as

$$
\sum_{k=1}^{K}\left(\frac{-P_{\max ^{2}} f_{p} h_{k} f_{k} B\left(z_{k}-\alpha_{k}^{0} t_{k}\right)}{\left(\alpha_{k}^{0} P_{\max } h_{k} f_{k}+\sigma^{2}+P_{\max } f_{p}\right)\left(\alpha_{k}^{0} P_{\max } h_{k} f_{k}+\sigma^{2}\right) \operatorname{In} 2}+F_{k}\left(\alpha_{k}^{0}\right)\right)+t_{\mathrm{e}} B \log _{2}\left(1+\frac{P_{\max } f_{p}}{\sigma^{2}}\right) \geq C_{\min }
$$

Accordingly, $\mathbf{P}_{3}$ can be transformed into the following subproblem, given by

$$
\begin{aligned}
& \mathrm{P}_{4}: \max _{t_{e},\left\{t_{k}\right\}_{k=1}^{K},\left\{z_{k}\right\}_{k=1}^{K}} \sum_{k=1}^{K} t_{k} B \log _{2}\left(1+\frac{\zeta z_{k} P_{\max } h_{k} g_{k}}{t_{k}\left(\eta P_{\max } f_{s}+\sigma^{2}\right)}\right) \\
& \text { s.t. } C 1^{\prime \prime \prime}: t_{k} B \log _{2}\left(1+\frac{\zeta z_{k} P_{\max } h_{k} g_{k}}{t_{k}\left(\eta P_{\max } f_{s}+\sigma^{2}\right)}\right) \geq C_{\min , k}, \forall k, \\
& \qquad \sum_{k=1}^{K} t_{k} B \log _{2}\left(1+\frac{-P_{\max }^{2} f_{p} h_{k} f_{k} B\left(z_{k}-\alpha_{k}^{0} t_{k}\right)}{\left(\alpha_{k}^{0} P_{\max } h_{k} f_{k}+\sigma^{2}+P_{\max } f_{p}\right)\left(\alpha_{k}^{0} P_{\max } h_{k} f_{k}+\sigma^{2}\right) \operatorname{In} 2}+F_{k}\left(\alpha_{k}^{0}\right)\right)+t_{e} B \log _{2}\left(1+\frac{P_{\max } f_{p}}{\sigma^{2}}\right) \geq C_{\min }, \\
& \quad C 2^{\prime \prime \prime}, C 5, C 7^{\prime} .
\end{aligned}
$$

Proposition 2. $\mathbf{P}_{4}$ is proved to be convex, which can be efficiently solved by using the existing convex optimization tools.

Proof. After using the first-order Taylor expression, the nonconvex QoS constraint for the PT's transmission in $\mathrm{Cl}^{\prime \prime \prime}$ can be turned into a linear constraint. Combining with
Proposition 1, $\mathbf{P}_{4}$ can be proved to be convex and can be efficiently solved by using the existing convex optimization tools. 
(1) Set the maximum tolerance $\varepsilon$ and the maximum number of iterations $I_{\max }$;

(2) Set the iteration index $i=1$ and the initial given values $\alpha_{k}^{0}, \forall k$;

(3) Based on Lemma 1, the optimal transmit power of the PT $P_{\mathrm{t}}^{*}$ is set as $P_{\max }$;

(4) repeat

(5) Solve the optimization problem $\mathbf{P}_{4}$ with given $\alpha_{k}^{0}, \forall k$, to obtain the optimal solutions, denoted by $t_{\mathrm{e}}^{*},\left\{t_{k}^{*}\right\}_{k=1}^{K},\left\{z_{k}^{*}\right\}_{k=1}^{K}$;

(6) Compute $\alpha_{k}^{*}$ as $\left(z_{k}^{*} / t_{k}^{*}\right), \forall k$;

(7) Compute the value of $R_{\text {tot }}^{s}$ based on (13);

(8) if $\left|\alpha_{k}^{*}-\alpha_{k}^{0}\right| \leq \varepsilon$ then

(9) Set Flag $=1$;

(10) else

(11) Set Flag $=0$ and $i=i+1$;

(12) Update $\alpha_{k}^{0}$ as $\alpha_{k}^{0}=\alpha_{k}^{*}, \forall k$;

(13) end if

(14) until Flag $=1$ or $i=I_{\max }$.

(15) Output $P_{\mathrm{t}}^{*}, t_{\mathrm{e}}^{*},\left\{t_{k}^{*}\right\}_{k=1}^{K},\left\{\alpha_{k}^{*}\right\}_{k=1}^{K}$ and $R_{\mathrm{tot}}^{s}$.

Algorithm 1: SCA based iterative algorithm.

3.3. Design of a SCA Based Iterative Algorithm. In this subsection, we propose a SCA based iterative algorithm to solve $\mathbf{P}_{3}$ efficiently. The detailed process of the proposed algorithm is shown in Algorithm 1.

As shown in Algorithm 1, in each iteration, we should use the existing convex tools, i.e., CVX, to optimally solve the subproblem $\mathbf{P}_{4}$ with given $\alpha_{k}^{0}, \forall k$. Then the optimal solutions to $\mathbf{P}_{4}$ are obtained, denoted by $t_{\mathrm{e}}^{*},\left\{t_{k}^{*}\right\}_{k=1}^{K},\left\{\alpha_{k}^{*}\right\}_{k=1}^{K}$, where $\alpha_{k}^{*}$ is computed as $\left(z_{k}^{*} / t_{k}^{*}\right), \forall k$. If the stop condition, namely, $\left|\alpha_{k}^{*}-\alpha_{k}^{0}\right| \leq \varepsilon$ with the maximum tolerance $\varepsilon$, is satisfied, then the solution to $\mathbf{P}_{3}$ is $t_{\mathrm{e}}^{*},\left\{t_{k}^{*}\right\}_{k=1}^{K},\left\{\alpha_{k}^{*}\right\}_{k=1}^{K}$. Otherwise, we should update the value of $\alpha_{k}^{0}$ as $\alpha_{k}^{*}$ and repeat the above steps until the stop condition is satisfied.

\section{Numerical Results}

In this section, both the effectiveness and the superiority of the proposed algorithm are verified via computer simulations. The key simulation parameters, unless otherwise specified, are provided in Table 1. Following [16], the parameters of the considered nonlinear $\mathrm{EH}$ model at the $k$-th ST are set as $a_{k}=2.463, d_{k}=1.635$, and $v_{k}=0.826, \forall k$. For the settings of all channels, we consider a standard channel fading model. Specifically, the channel gain of the PT-PR link is modeled by $f_{p}=f_{p}^{\prime} D_{p}^{-\beta}$, where $f_{p}^{\prime}$ denotes the smallscale fading of the PT-PR link, $D_{p}$ is the distance from the $\mathrm{PT}$ to the PR, and $\beta$ denotes the path loss exponent. The channel gain of the PT- $k$-th ST link is given by $g_{k}=g_{k}^{\prime} D_{1, k}^{-\beta}, \forall k$, where $g_{k}^{\prime}$ and $D_{1, k}$ are the small-scale fading and the distance from the PT to the $k$-th ST, respectively. The channel gain of the $k$-th ST-SR link is modeled by $h_{k}=h_{k}^{\prime} D_{2, k}^{-\beta}, \forall k$, where $h_{k}^{\prime}$ and $D_{2, k}$ are the small-scale fading and the distance from the $k$-th ST to the SR, respectively. The channel gain of the $k$-th ST-PR link is $f_{k}=f_{k}^{\prime} D_{3, k}^{-\beta}, \forall k$ with the small-scale fading $f_{k}^{\prime}$ and the distance $D_{3, k}$. The channel gain of the PT-SR link is modeled by $f_{s}=f_{s}^{\prime} D_{s}^{-\beta}, \forall k$, with the small-scale fading $f_{s}^{\prime}$ and the distance $D_{s}$. In the simulations, we set $\beta=2.7, D_{p}=30 \mathrm{~m}, D_{s}=30 \mathrm{~m}, D_{1,1}=12 \mathrm{~m}$, $D_{1,2}=10 \mathrm{~m}, D_{1,3}=15 \mathrm{~m}, D_{1,4}=13 \mathrm{~m}, D_{2,1}=20 \mathrm{~m}, D_{2,2}=$
$15 \mathrm{~m}, D_{2,3}=20 \mathrm{~m}, D_{2,4}=15 \mathrm{~m}, D_{3,1}=25 \mathrm{~m}, D_{3,2}=30 \mathrm{~m}$, $D_{3,3}=30 \mathrm{~m}$, and $D_{3,4}=30 \mathrm{~m}$.

Figure 2 demonstrates the convergence of Algorithm 1, where different settings of $C_{s, \min }$ are considered and $C_{\min ^{1}}=C_{\min ^{2}}=C_{\min ^{3}}=C_{\min ^{4}}=C_{s, \min }$. We set $C_{s, \min }$ as 10 bits, 20 bits, and 30 bits. It can be observed that the proposed algorithm in Algorithm 1 can always converge to a certain value after only a few iterations, i.e., 3 iterations. This indicates that the proposed algorithm is convergent and computationally efficient. Besides, it can also be seen that a larger $C_{s, \text { min }}$ brings a lower total throughput of all STs. This is because a larger $C_{s, \text { min }}$ means a higher QoS requirement for the ST's transmission, and more resources will be allocated to the STs with worse channels, leading to a reduction in the total throughput of all STs.

Figure 3 plots the total throughput of all STs versus the minimum required throughput for each $\mathrm{ST} C_{s, \min }$, and $C_{s, \text { min }}$ is varied from 5 bits to 30 bits. In order to illustrate the advantages of the proposed scheme, we compare the performance achieved by the proposed scheme with the fixed scheme, where the power reflection coefficient of each ST is fixed as $0.5,0.8$, and 0.9 , respectively. As shown in this figure, the total throughput of all STs decreases with the increase of $C_{s \text {, min }}$, since a larger $C_{s, \text { min }}$ brings a higher QoS requirement for the ST's transmission, and more resources will be allocated to the STs with worse channels, leading to a reduction in the total throughput of all STs. By comparisons, we can see that the total throughput of all STs under the proposed scheme is higher than that under the fixed scheme, as the proposed scheme provides more flexibilities to utilize resources efficiently for maximizing the total throughput of all STs. This also demonstrates the superiority of the proposed scheme.

Figure 4 shows total throughput of all STs versus the minimum SINR threshold required for decoding $x_{p}, \gamma_{\mathrm{th}}$, where $\gamma_{\text {th }}$ ranges from 20 to 100 . The power reflection coefficient of each SN under the fixed scheme is set as $0.5,0.8$, and 0.9. It can be observed from this figure that the total throughput of all STs decreases when $\gamma_{\text {th }}$ increases. This is because a higher $\gamma_{\text {th }}$ brings a higher requirement for 
TABLE 1: Key simulation settings.

\begin{tabular}{|c|c|c|}
\hline Parameter & Notation & Value \\
\hline The entire time block & $T$ & 1 second \\
\hline The communication bandwidth & $B$ & $100 \mathrm{kHz}$ \\
\hline The constant circuit power consumption for BackCom at the $k$-th ST & $P_{\mathrm{b}, k}$ & $10 \mu \mathrm{W}$ \\
\hline The maximum transmit power at the $\mathrm{PT}$ & $P_{\max }^{\mathrm{D}, \Lambda}$ & $1 \mathrm{~W}$ \\
\hline The performance gap reflecting the real modulation for BackCom & $\xi$ & $-15 \mathrm{~dB}$ \\
\hline The noise power & $\sigma^{2}$ & $-60 \mathrm{dBm}$ \\
\hline The number of STs & K & 4 \\
\hline The minimum required throughput of the $k$-th ST & $C_{\min , k}$ & 10 bits \\
\hline The minimum required throughput of the $\mathrm{PT}$ & $C_{\min }$ & 100 bits \\
\hline The threshold required for decoding $x_{p}$ & $\gamma_{\text {th }}$ & 20 \\
\hline
\end{tabular}

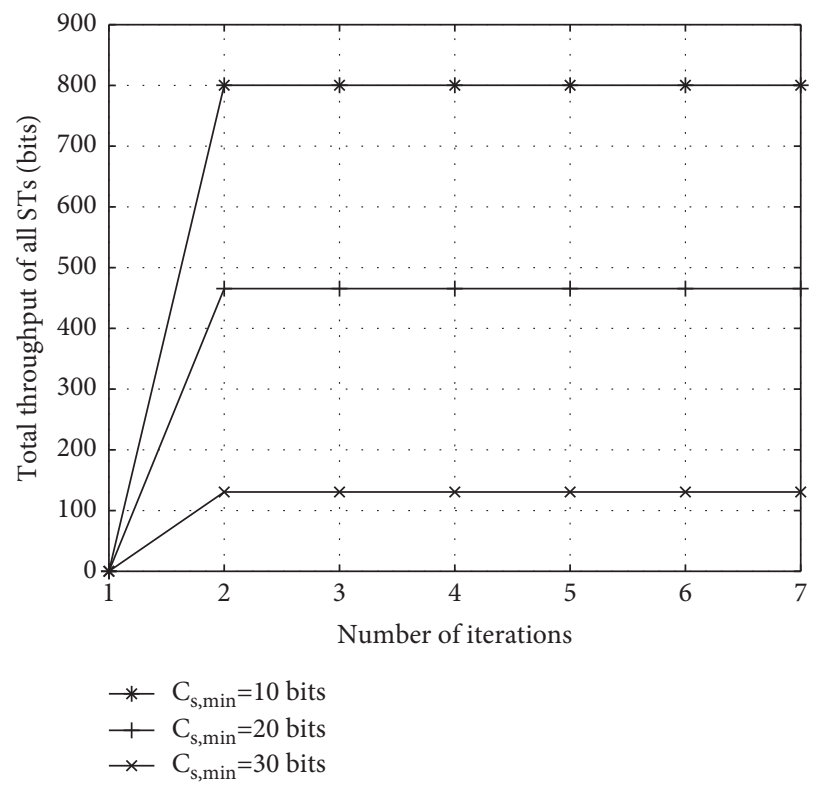

FIgURE 2: The convergence of Algorithm 1 under different settings of $C_{s, \min }$, where $C_{\min ^{1}}=C_{\min ^{2}}=C_{\min ^{3}}=C_{\min ^{4}}=C_{s, \min }$.

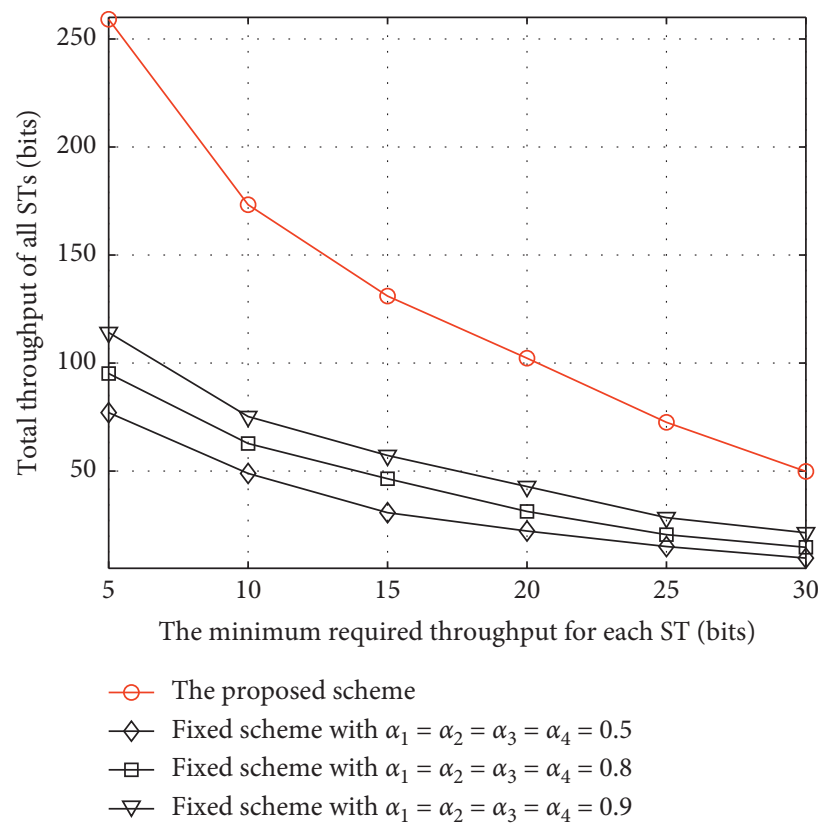

FIgURE 3: Total throughput of all STs versus the minimum required throughput for each ST. 


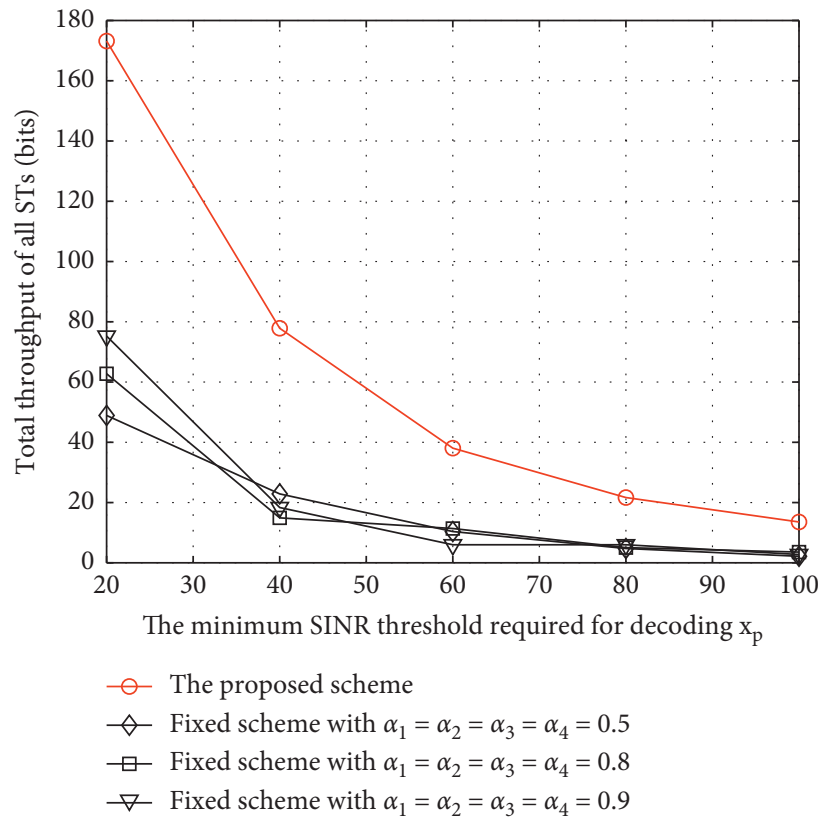

FIGURE 4: Total throughput of all STs versus the minimum SINR threshold required for decoding $x_{p}$.

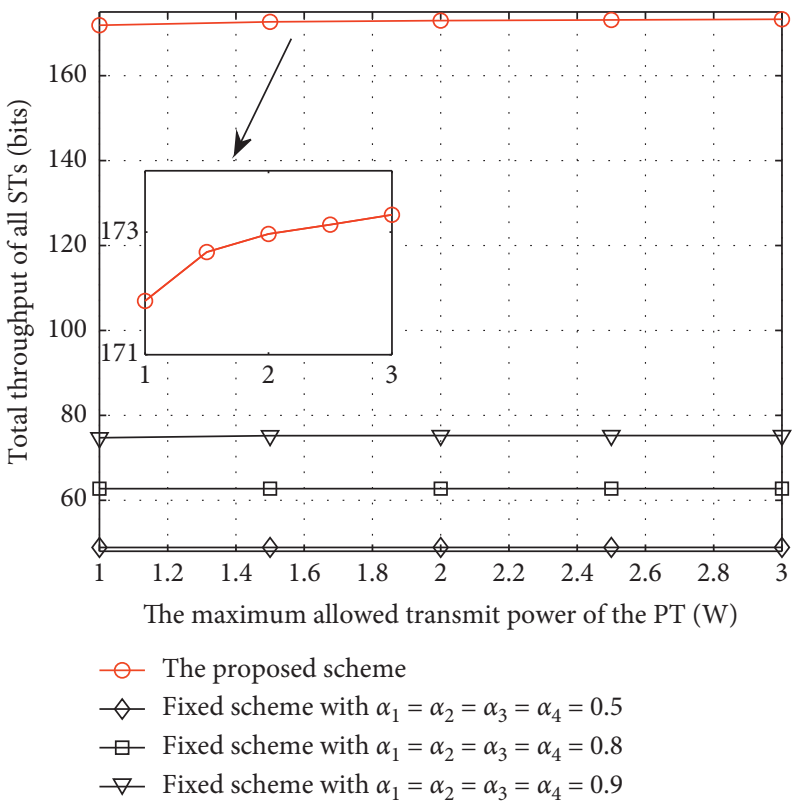

FIGURE 5: Total throughput of all STs versus the maximum allowed transmit power at the PT.

decoding $x_{p}$, leading to a reduction in the total throughput of all STs. By comparisons, we can observe that the proposed scheme outperforms the other schemes in terms of the total throughput of all STs, which illustrates the advantages of the proposed scheme.

Figure 5 shows the total throughput of all STs versus the maximum allowed transmit power at the PT $P_{\max }$ under different schemes. Here, $P_{\max }$ varies from $1 \mathrm{~W}$ to $3 \mathrm{~W}$. It can be observed that the total throughput of all STs under all the schemes increase with the increase of $P_{\max }$. Based on Lemma 1, the optimal transmit power of the PT is determined by
$P_{\text {max }}$, and a higher transmit power of the PT allows STs to harvest more energy for supporting the energy consumption of BackCom and to backscatter signals with a higher power, resulting in an improvement for the total throughput of all STs. Besides, we also observe that the total throughput of all STs under the proposed scheme is the highest among these schemes, which also demonstrates the superiority of the proposed scheme in terms of total throughput of all STs. 


\section{Conclusions}

In this paper, we have studied the throughput maximization for the BackCom based cognitive network while considering a nonlinear EH model. Specifically, we have formulated an optimization problem to maximize the total throughput of all STs by jointly optimizing the $\mathrm{EH}$ time, the transmit power of the PT, the BackCom time, and the power reflection coefficient of each ST under the QoS, energy causality, latency, transmit power, and power reflection coefficient constraints. In order to solve the nonconvex problem, we first determined the optimal transmit power of the PT by using the properties of the objective function and then proposed a SCA based iterative algorithm to obtain the proposed scheme. The simulation results verified the quick convergence of the proposed algorithm and showed that the proposed scheme outperforms the other schemes in terms of total throughput of all STs.

\section{Appendix}

\section{A. Proof of Lemma 1}

Let $C_{k}\left(P_{t}\right)=t_{k} B \log _{2}\left(1+\left(\xi \alpha_{k} P_{t} h_{k} g_{k} / \eta P_{t} f_{s}+\sigma^{2}\right)\right) \quad$ and $R_{\text {tot }}^{s}=\sum_{k=1}^{K} C_{k}\left(P_{\mathrm{t}}\right)$. By taking the first-order derivative of $R_{\text {tot }}^{s}$ with respect to $P_{\mathrm{t}}$, we have

$$
\frac{\partial R_{t o t}^{s}}{\partial P_{t}}=\sum_{k=1}^{K} \frac{\partial C_{k}\left(P_{t}\right)}{\partial P_{t}}=\sum_{k=1}^{K} \frac{t_{k} B \xi \alpha_{k} h_{k} g_{k} \sigma^{2}}{\left(\eta P_{t} f_{s}+\sigma^{2}\right)\left(\eta P_{t} f_{s}+\sigma^{2}+\xi \alpha_{k} h_{k} g_{k} P_{t}\right) \operatorname{In} 2}
$$

Since $\left(\partial R_{\text {tot }}^{s} / \partial P_{\mathrm{t}}\right)>0$ always holds, $R_{\text {tot }}^{s}$ is a monotone increasing function with respect to $P_{\mathrm{t}}$. That is to say, a larger $P_{\mathrm{t}}$ brings a larger $R_{\text {tot }}^{s}$. In order to maximize $R_{\text {tot }}^{s}$, the optimal transmit power of the PT, denoted by $P_{\mathrm{t}}^{*}$, should equal the maximum within its feasible region.

By observing the constraints $\mathrm{C} 1, \mathrm{C} 2, \mathrm{C} 3$, and $\mathrm{C} 4$, we can find that the lower bound of $P_{\mathrm{t}}$ is determined by $\mathrm{C} 1, \mathrm{C} 2$, and C4 while the upper bound of $P_{\mathrm{t}}$ is always $P_{\max }$. The reasons are as follows. As for $\mathrm{C} 1$, similar to $R_{\text {tot }}^{s}$, we can prove that the functions $C_{k}\left(P_{\mathrm{t}}\right)$ and $\sum_{k=1}^{K} t_{k} B \log _{2}\left(1+\left(P_{\mathrm{t}} f_{p} / \alpha_{k} P_{\mathrm{t}} h_{k} f_{k}+\right.\right.$ $\left.\left.\sigma^{2}\right)\right)+t_{\mathrm{e}} B \log _{2}\left(1+\left(P_{\mathrm{t}} f_{p} / \sigma^{2}\right)\right)$ are monotone increasing functions with respect to $P_{\mathrm{t}}$. The proof process is omitted here for brevity. Therefore, $\mathrm{C} 1$ determines a lower bound of $P_{\mathrm{t}}$.

As for $\mathrm{C} 2$, since the harvested power of the $\mathrm{EH}$ circuit increases with the increase of the input power and then converges to the maximum value when the input power is large enough, $f_{k}(x)$ is a monotone increasing function with respect to $x$. That is, the right side of $\mathrm{C} 2$ is also a monotone increasing function with respect to $P_{\mathrm{t}}$, and $P_{\mathrm{t}}$ determines another lower bound of $P_{\mathrm{t}}$.

As for C4, based on (6), we can transform C4 as $P_{\mathrm{t}} \geq\left(\gamma_{\mathrm{th}} \sigma^{2} / f_{s}-\gamma_{\mathrm{th}} \alpha_{k} h_{k} g_{k}\right), \forall k$, which is also a lower bound of $P_{\mathrm{t}}$.

Therefore, the upper bound of $P_{\mathrm{t}}$ is only determined by $P_{\max }$, and the optimal transmit power of the PT is given by $P_{\mathrm{t}}^{*}=P_{\max }$. Then, the proof of Lemma 1 is complete.

\section{B. Proof of Proposition 1}

After carefully analyzing $\mathbf{P}_{3}$, it is not hard to conclude that both constraints $\mathrm{C} 5$ and $\mathrm{C} 7^{\prime}$ are linear constraints. Thus, $\mathbf{P}_{3}$ is convex if and only if the objective function is a concave function and constraints $\mathrm{C} 1^{\prime \prime}$ and $\mathrm{C} 2{ }^{\prime \prime}$ are convex.

For the objective function, using the fact that the perspective function can preserve convexity, we can find that the convexity of the function $t_{k} B \log _{2}\left(1+\left(\xi z_{k} P_{\max }\right.\right.$ $\left.\left.h_{k} g_{k} / t_{k}\left(\eta P_{\max } f_{s}+\sigma^{2}\right)\right)\right)$ is the same as that of the function $\log _{2}\left(1+\left(\xi z_{k} P_{\max } h_{k} g_{k} / \eta P_{\max } f_{s}+\sigma^{2}\right)\right)$. Since $\log _{2}(1+$ $\left.\left(\xi z_{k} P_{\max } h_{k} g_{k} / \eta P_{\max } f_{s}+\sigma^{2}\right)\right)$ is a concave function with respect to $z_{k}, \quad t_{k} B \log _{2}\left(1+\left(\xi z_{k} P_{\max } h_{k} g_{k} / t_{k}(\eta P\right.\right.$ $\left.\left.\max f_{s}+\sigma^{2}\right)\right)$ ) is a concave function jointly with respect to $z_{k}$ and $t_{k}$. Thus, the objective function is a concave function.

For the QoS constraint for the ST's transmission in $\mathrm{C1}^{\prime \prime}$, since $t_{k} B \log _{2}\left(1+\left(\xi z_{k} P_{\max } h_{k} g_{k} / t_{k}\left(\eta P_{\max } f_{s}+\sigma^{2}\right)\right)\right)$ is a concave function jointly with respect to $z_{k}$ and $t_{k}$, the QoS constraint for the ST's transmission is convex. For the QoS constraint for the PT's transmission in $\mathrm{Cl}^{\prime \prime}, t_{k} B \log _{2}(1+$ $\left.\left(t_{k} P_{\max } f_{p} / z_{k} P_{\max } h_{k} f_{k}+\sigma^{2} t_{k}\right)\right)$ is neither convex nor concave, leading to the nonconvex QoS constraint for the PT's transmission and the nonconvex constraint $\mathrm{C} 1^{\prime \prime}$.

For the constraint $\mathrm{C} 2{ }^{\prime \prime}$, its convexity depends on the convexity of $t_{k} f_{k}\left(\left(t_{k}-z_{k}\right) P_{\max } h_{k} / t_{k}\right)$. Based on the perspective function, the convexity of $t_{k} f_{k}\left(\left(t_{k}-z_{k}\right) P_{\max } h_{k} / t_{k}\right)$ is the same as that of $f_{k}\left(\left(1-z_{k}\right) P_{\max } h_{k}\right)$. By taking the firstorder derivative of $f_{k}\left(\left(1-z_{k}\right) P_{\max } h_{k}\right)$ with respect to $\left(1-z_{k}\right) P_{\max } h_{k}$, we have

$$
\frac{\partial f_{k}\left(\left(1-z_{k}\right) P_{\max } h_{k}\right)}{\partial\left(1-z_{k}\right) P_{\max } h_{k}}=\frac{a_{k} v_{k}-d_{k}}{\left(\left(1-z_{k}\right) P_{\max } h_{k}+v_{k}\right)^{2}} .
$$

Since the harvested power of the $\mathrm{EH}$ circuit increases with the input power, $\left(\partial f_{k}\left(\left(1-z_{k}\right) P_{\max } h_{k}\right) / \partial\left(1-z_{k}\right)\right.$ $\left.P_{\max } h_{k}\right)$ should be always larger than or equal to 0 . Therefore, $a_{k} v_{k}-d_{k} \geq 0$ always holds. Then, we take the second-order derivative of $f_{k}\left(\left(1-z_{k}\right) P_{\max } h_{k}\right)$ with respect to $z_{k}$; we have

$$
\frac{\partial^{2} f_{k}\left(\left(1-z_{k}\right) P_{\max } h_{k}\right)}{\partial z_{k}^{2}}=\frac{-2\left(P_{\max } h_{k}\right)^{2}\left(a_{k} v_{k}-d_{k}\right)}{\left(\left(1-z_{k}\right) P_{\max } h_{k}+v_{k}\right)^{3}}
$$

According to $a_{k} v_{k}-d_{k} \geq 0$, we have $\partial^{2} f_{k}\left(\left(1-z_{k}\right) P_{\max } h_{k}\right) / \partial z_{k}^{2} \leq 0$. Therefore, $f_{k}\left(\left(1-z_{k}\right) P_{\max } h_{k}\right)$ is a concave function with respect to $z_{k}$. Correspondingly, $t_{k} f_{k}\left(\left(\left(t_{k}-z_{k}\right) P_{\max } h_{k} / t_{k}\right)\right)$ is also a concave function jointly with respect to $z_{k}$ and $t_{k}$, and the constraint $\mathrm{C}^{\prime \prime}$ is convex. 
Based on the above analysis, Proposition 1 is obtained, and the proof is complete.

\section{Data Availability}

The simulation data used to support the findings of this study are included within the article. The MATLAB code used to support the findings of this study is available from the corresponding author upon request.

\section{Conflicts of Interest}

The author declares that there are no conflicts of interest.

\section{Acknowledgments}

This work was supported by the University Key Scientific Research Project of Henan Province (no. 22A520052).

\section{References}

[1] A. Al-Fuqaha, M. Guizani, M. Mohammadi, M. Aledhari, and M. Ayyash, "Internet of things: a survey on enabling technologies, protocols, and applications," IEEE Communications Surveys \& Tutorials, vol. 17, no. 4, pp. 2347-2376, 2015.

[2] J. M. Peha, "Sharing spectrum through spectrum policy reform and cognitive radio," Proceedings of the IEEE, vol. 97, no. 4, pp. 708-719, 2009.

[3] N. Van Huynh, D. T. Hoang, X. Lu, D. Niyato, P. Wang, and D. I. Kim, "Ambient backscatter communications: a contemporary survey," IEEE Communications Surveys \& Tutorials, vol. 20, no. 4, pp. 2889-2922, 2018.

[4] Y. Ye, L. Shi, X. Chu, and G. Lu, "On the outage performance of ambient backscatter communications," IEEE Internet of Things Journal, vol. 7, no. 8, pp. 7265-7278, 2020.

[5] L. Shi, R. Q. Hu, Y. Ye, and H. Zhang, "Modeling and performance analysis for ambient backscattering underlaying cellular networks," IEEE Transactions on Vehicular Technology, vol. 69, no. 6, pp. 6563-6577, 2020.

[6] X. Kang, Y.-C. Liang, and J. Yang, "Riding on the primary: a new spectrum sharing paradigm for wireless-powered iot devices," in Proceedings of the 2017 IEEE International Conference on Communications (ICC), pp. 1-6, Paris, France, May 2017.

[7] Y. Ye, L. Shi, R. Qingyang Hu, and G. Lu, "Energy-efficient resource allocation for wirelessly powered backscatter communications," IEEE Communications Letters, vol. 23, no. 8, pp. 1418-1422, 2019.

[8] J. Wang, H.-T. Ye, X. Kang, S. Sun, and Y.-C. Liang, "Cognitive backscatter noma networks with multi-slot energy causality," IEEE Communications Letters, vol. 24, no. 12, pp. 2854-2858, 2020.

[9] S. Xiao, H. Guo, and Y.-C. Liang, "Resource allocation for fullduplex-enabled cognitive backscatter networks," IEEE Transactions on Wireless Communications, vol. 18, no. 6, pp. 3222-3235, 2019.

[10] D. T. Hoang, D. Niyato, P. Wang, and D. I. Kim, "Optimal time sharing in rf-powered backscatter cognitive radio networks," in Proceedings of the 2017 IEEE International Conference on Communications (ICC), pp. 1-6, Paris, France, May 2017.

[11] R. Kishore, S. Gurugopinath, P. C. Sofotasios, S. Muhaidat, and N. Al-Dhahir, "Opportunistic ambient backscatter communication in RF-powered cognitive radio networks," IEEE Transactions on Cognitive Communications and Networking, vol. 5, no. 2, pp. 413-426, 2019.

[12] D. T. Hoang, D. Niyato, P. Wang, D. I. Kim, and Z. Han, "Ambient backscatter: a new approach to improve network performance for RF-powered cognitive radio networks," IEEE Transactions on Communications, vol. 65, no. 9, pp. 36593674, 2017.

[13] T. Wang, G. Lu, Y. Ye, and Y. Ren, "Dynamic power splitting strategy for SWIPT based two-way multiplicative AF relay networks with nonlinear energy harvesting model," Wireless Communications and Mobile Computing, vol. 2018, no. 1, pp. 1-9, 2018.

[14] E. Boshkovska, D. W. K. Ng, N. Zlatanov, A. Koelpin, and R. Schober, "Robust resource allocation for mimo wireless powered communication networks based on a non-linear eh model," IEEE Transactions on Communications, vol. 65, no. 5, pp. 1984-1999, 2017.

[15] H. Yang, Y. Ye, X. Chu, and M. Dong, "Resource and power allocation in SWIPT-enabled device-to-device communications based on a nonlinear energy harvesting model," IEEE Internet of Things Journal, vol. 7, no. 11, pp. 10813-10825, 2020.

[16] Y. Chen, N. Zhao, and M.-S. Alouini, "Wireless energy harvesting using signals from multiple fading channels," IEEE Transactions on Communications, vol. 65, no. 11, pp. 50275039, 2017.

[17] Y. Liu, Y. Ye, H. Ding, F. Gao, and H. Yang, "Outage performance analysis for SWIPT-based incremental cooperative NOMA networks with non-linear harvester," IEEE Communications Letters, vol. 24, no. 2, pp. 287-291, 2020.

[18] L. Shi, Y. Ye, R. Q. Hu, and H. Zhang, "Energy efficiency maximization for SWIPT enabled two-way DF relaying," IEEE Signal Processing Letters, vol. 26, no. 5, pp. 755-759, 2019.

[19] Y. Ye, L. Shi, X. Chu, and G. Lu, "Throughput fairness guarantee in wireless powered backscatter communications with HTT," IEEE Wireless Communications Letters, vol. 10, no. 3, pp. 449-453, 2021.

[20] S. H. Kim and D. I. Kim, "Hybrid backscatter communication for wireless-powered heterogeneous networks," IEEE Transactions on Wireless Communications, vol. 16, no. 10, pp. 6557-6570, 2017.

[21] H. Yang, Y. Ye, X. Chu, and S. Sun, "Energy efficiency maximization for UAV-enabled hybrid backscatter-harvestthen-transmit communications," IEEE Transactions on Wireless Communications, vol. 1, 2021. 